\title{
OIP5-AS1 contributes to the development in endometrial carcinoma cells by targeting miR-152-3p to up-regulate SLC7A5
}

Minglin Liang, Hongbo Wang, Cong Liu, Tao Lei and Jie Min*

\begin{abstract}
Background: Endometrial carcinoma (EC) is one common gynecological tumor, threatening physical and psychological health of females. Huge amount of essays indicated that long non-coding RNAs (IncRNAs) were widely reported to serve as a crucial regulator in the biological movements among multiple carcinomas, including EC.

Methods: RT-qPCR was implemented to detect the expression of target genes. Loss/gain-of-function experiments certified the impacts of OIP5-AS1 and miR-152-3p on EC cell progression.

Results: Data of this research suggested that powerful expression of OIP5-AS1 was discovered in EC cell lines. Loss/ gain-of-function assays inferred that OIP5-AS1 promoted proliferative, migratory and invasive abilities, and EpithelialMesenchymal Transition (EMT). In addition, we identified miR-152-3p expression was negatively modulated by OIP5-AS1. OIP5-AS1 accelerated the development of EC cells via downregulating miR-152-3p expression. SLC7A5 was selected out as a downstream target of miR-152-3p. The competing relationship between OIP5-AS1 and SLC7A5 was corroborated by luciferase reporter assay. Eventually, the results of rescue assays indicated that SLC7A5 overexpression could restore the impacts of OIP5-AS1 ablation on the progression of EC cells.
\end{abstract}

Conclusion: Our research confirmed that OIP5-AS1 propeled the development of EC cells through targeting miR152-3p/SLC7A5. OIP5-AS1 could be utilized as a target for EC treatment.

Keywords: OIP5-AS1, miR-152-3p, SLC7A5, Endometrial carcinoma

\section{Background}

Endometrial carcinoma (EC) is considered as one of the most widespread gynecological malignancies in European Union, with more than 73,000 cases diagnosed in $2020[1]$. It is first and foremost to choose surgical operation as prioritized therapeutic methods for EC treatment. With the consolidation of adjuvant chemotherapy and radiotherapy, the survival rate is improved and progression is delayed in EC [2, 3]. In the late decades, although great progress has been made in therapies for EC, the

*Correspondence: 691055202@qq.com

Department of Obstetrics and Gynecology, Union Hospital, Tongji Medical College, Huazhong Univercity of Science and Technology, No. 2177 Liberation Avenue, Wuhan 430022, China prognosis of EC patients still remains poor [4]. The recurrence rate is high due to the metastasis in the endstage of EC. Therefore, it is vital to probe into the pathology mechanism of EC and ascertain a remedy target to improve the treatment.

LncRNAs are a cluster of transcripts with over 200 bases in length and without the ability to translate proteins [5]. Emerging evidence has attested the importance of lncRNAs in the process of various cancers. For example, LINC01170 accelerated the growth of EC via activation of AKT pathway [6]. PVT1 acted as an oncogene in ovarian cancer through enhancing SOX2 [7]. The regulatory role of OIP5-AS1 was studied in osteosarcoma [8], lung cancer [9] and colorectal cancer [10]. Nevertheless, original author(s) and the source, provide a link to the Creative Commons licence, and indicate if changes were made. The images or other third party material in this article are included in the article's Creative Commons licence, unless indicated otherwise in a credit line to the material. If material is not included in the article's Creative Commons licence and your intended use is not permitted by statutory regulation or exceeds the permitted use, you will need to obtain permission directly from the copyright holder. To view a copy of this licence, visit http://creativecommons.org/licenses/by/4.0/. The Creative Commons Public Domain Dedication waiver (http://creativeco mmons.org/publicdomain/zero/1.0/) applies to the data made available in this article, unless otherwise stated in a credit line to the data. 
the link between OIP5-AS1 and EC still remains to be explored.

MicroRNAs (miRNAs) are defined as the RNAs featuring about 18-25 nucleotides in length and inability to code proteins, and they can modulate target gene expression through interacting with the 3 ' untranslated region (UTR) of the mRNAs, which are their downstream targets [11]. Accumulating researches indicated that miRNAs were active regulators of the proliferation, migration and chemoresistance in numerous cancers. For instance, miR-21 acted as an oncogene in tongue squamous cell by promoting proliferation and invasion in vitro [12]. MiR630 suppressed metastasis in esophageal squamous cell carcinoma [13]. MiR-135b accelerated proliferation of EC through targeting FOXO1 [14]. MiR-152-3p modulated progression of prostate cancer by inhibiting KLF4 synergistically with miR-148-3p. However, the detailed function of miR-152-3p has not been explained thoroughly in EC.

Our main aim was to probe into the mechanisms and functions of OIP5-AS1 in EC cells and the interaction between miR-152-3p and OIP5-AS1 in our study. Furthermore, the underlying targets of miR-152-5p would also be investigated.

\section{Materials and methods}

\section{Cell lines and tissue samples}

EC cell lines (Ishikawa and ECC), and the normal cell line (SHT290) were procured from the Cell Resource Center of Chinese Academy of Medical Sciences (Beijing, China), then cultivated in the Dulbecco's modified Eagle's medium (DMEM; Gibco, Grand Island, NY). Cell culture was implemented with $10 \%$ fetal bovine serum (FBS; Gibco) as medium supplement, in the $95 \%$ air $/ 5 \% \mathrm{CO}_{2}$ incubator at $37^{\circ} \mathrm{C}$.

\section{RNA extraction and real-time quantitative PCR (RT-qPCR)}

The total cellular RNAs were severally extracted on the basis of the protocol of TRIzol reagent (Invitrogen, Carlsbad, CA). $3 \mu \mathrm{g}$ of total RNAs were converted into the cDNA by applying the Reverse Transcription Kit (Toyobo, Osaka, Japan). Gene expression was quantified by ABI Step-One Plus Real-Time PCR System (Applied Biosystems, Foster City, CA) with the SYBR ${ }^{\circledR}$ Premix Ex $\mathrm{Taq}^{\mathrm{TM}}$ II (Takara). As for Data calculation, $2^{-\Delta \Delta \mathrm{CT}}$ method was used, with GAPDH and U6 as controls.

\section{Cell transfection}

The short hairpin RNAs (shRNAs) against OIP5-AS1 were constructed by Genepharma (Shanghai, China) for gene silencing analysis, as well as its control, sh-NC. The pcDNA3.1/OIP5-AS1, pcDNA3.1/SLC7A5 and the control pcDNA3.1 vectors were commercially obtained from Ribobio (Guangzhou, China) to up-regulate target genes. Besides, the miR-152-3p mimics/inhibitor, as well as the corresponding negative control (NC mimics/ inhibitor) were designed at Genepharma. Plasmids were transfected into EC cell for $48 \mathrm{~h}$ via Lipofectamine 2000 (Invitrogen).

\section{Colony formation}

Clonogenic cell samples were cultivated as 500 cells per well into the 6 -well plates for 14 days, followed by the treatment with the $0.5 \%$ crystal violet staining solution in $4 \%$ paraformaldehyde. Clones were determined and counted.

\section{Flow cytometry analysis of apoptosis}

Flow cytometry analysis was performed for the examination of cell apoptosis. The cells were stained in Annexin V-labeled with 7AAD and PE solution (BD Biosciences, San Jose, CA) for a quarter of an hour in the dark. After being rinsed in the pre-chilled phosphate-buffered saline (PBS) and double-staining, cell samples were assayed by FACS cytometry (BD Biosciences) finally.

\section{Western blot}

30-50 mg of the cellular total proteins were subjected to RIPA lysis buffer for isolation and then to $12 \%$ SDS-PAGE gel for separation. Subsequently, the separated protein samples were electro-blotted onto the PVDF membranes (Millipore, Billerica, MA), and then treated with the 5\% skimmed milk solution for blocking. Samples were cultivated with the primary antibodies against the loading control GAPDH and Bcl-2, Bax, Cleaved caspase-3, Total caspase-3, as well as four EMT markers, slug, Vimentin, N-cadherin, E-cadherin (at 1: 2000 dilution, Abcam, Cambridge, MA) for a whole night. Followed by rinsing in the Tris-buffered saline (TBST), samples were incubated with the secondary antibodies (at 1: 5000; Abcam) for $2 \mathrm{~h}$. Blots were finally detected by Immobilon ${ }^{\circledR}$ Western Horseradish peroxidase substrate (Millipore).

\section{Transwell assays}

$6 \times 10^{4}$ cell samples in the $100 \mathrm{ml}$ of medium free of FBS were placed into the upper part of the Transwell chamber (Corning Life Sciences, Corning, NY). $600 \mathrm{ml}$ of conditioned medium was placed into the lower chamber. Followed by $24 \mathrm{~h}$ of incubation at $37{ }^{\circ} \mathrm{C}$, migrating cell samples were fixed for $15 \mathrm{~min}$ and treated with $0.5 \%$ crystal violet staining for $30 \mathrm{~min}$. The up-right fluorescence microscope $(200 \times)$ was applied for counting. Cell invasion was analyzed with Transwell chamber coated by the Matrigel membrane (BD Biosciences). 


\section{FISH assay}

Cell samples were first fixed for $15 \mathrm{~min}$, then rinsed in PBS and air-dried. $40 \mathrm{~nm}$ of the FISH probe synthesized for OIP5-AS1 was procured from Ribobio and mixed with the cell samples in the hybridization buffer. As for the counterstaining of nucleus, Hoechst solution was adopted. The final images were captured using the fluorescence microscope.

\section{RNA pull down}

The assay was conducted by employing the Pierce Magnetic RNA-Protein Pull-Down Kit (Thermo Fisher Scientific, Waltham, MA) in light of the user manual. The wild-type (WT) or mutated (Mut) miR-152-3p sequences with the OIP5-AS1 binding sites were constructed, followed by the biotinylation into Bio-miR152-3p-WT/Mut probes. The cells, after being lysed, were subjected to the $1 \mathrm{~h}$-incubation with the probes and streptavidin agarose magnetic beads. After RNA extraction and purification, RT-qPCR was finally conducted for determining OIP5-AS1 enrichment in pull-downs.

\section{Dual-luciferase reporter assays}

OIP5-AS1-WT/Mut or SLC7A5-WT/Mut fragments covering the miR-152-3p interacting sequences were constructed and subjected to the subcloning into the pmirGLO Dual-Luciferase Expression Vector (Promega, Madison, WI). Afterwards, the reporter vectors were subjected to the co-transfection into EC cells along with the specific plasmids for $48 \mathrm{~h}$. Dual-Luciferase Reporter Assay System (Promega) was employed for luciferase activity.

\section{RNA immunoprecipitation (RIP)}

The assay was implemented on a basis of the instruction of EZ-Magna RIP RNA Binding Protein Immunoprecipitation Kit (Millipore). The cultured cell samples were lysed in the RIP lysis buffer, then collected and treated with magnetic beads bound to the antibodies of Ago2 or IgG (Millipore) in RIP buffer. Following RNA extraction, the purified RNAs were assayed by RT-qPCR.

\section{Statistical analysis}

All assays were subjected to independent bio-repetition in triplicate. All the experimental results were represented as the mean \pm standard deviation (SD). Data analysis was developed by Student's t-test and one-way or two-way analysis of variance (ANOVA) employing the
Prism 5.0 (GraphPad Software Inc., La Jolla, CA). Significant level was set as the p-value less than 0.05 .

\section{Results}

OIP5-AS1 is up-regulated in EC cells and boosts the development of EC cells

At the beginning, we unveiled the potential role of OIP5AS1 in EC. We observed from RT-qPCR a high expression of OIP5-AS1 in Ishikawa and ECC cells compared with SHT290 (Fig. 1A). Next, RT-qPCR testing was implemented for the detection of the knockdown efficacy of sh-OIP5-AS1\#1/2 in Ishikawa and ECC cells (Fig. 1B). Furthermore, we performed loss-of-functional assays to confirm the influences of OIP5-AS1 on Ishikawa and ECC cells. Data from colony formation assays showcased that OIP5-AS1 silence decreased proliferation abilities in colony formation assays (Fig. 1C). On the contrary, OIP5-AS1 knockdown increased the apoptosis rate prominently (Fig. 1D). Western blot measured the associated proteins of apoptosis. According to the results, Cleaved caspase- 3 and Bax proteins were elevated while Bcl-2 protein was decreased by OIP5-AS1 silencing (Fig. 1E). Results of transwell exhibited that the abilities to migrate and invade were weakened by OIP5-AS1 down-regulation (Fig. 1F, G). The EMT markers were measured via the implementation of western blot. Consequences displayed that E-cadherin was up-regulated while $\mathrm{N}$-cadherin, vimentin, as well as Slug were lessened by OIP5-AS1 silence (Fig. 1H). To further verify the effect of OIP5-AS1, we subsequently conducted gain-of-functional assays in Ishikawa and ECC cells, with detecting the overexpression efficiency of pcDNA3.1/OIP5-AS1 first (Additional file 1: Fig. S1A). The results of functional assays and western blot showed that overexpressed OIP5-AS1 enhanced the development, and suppressed apoptosis of Ishikawa and ECC cells (Additional file 1: Fig. S1B-F). Taken together, OIP5-AS1 is high-expressed in EC cells, and OIP5-AS1 promotes the process of EC cells.

\section{OIP5-AS1 negatively regulates miR-152-3p in EC cells}

For further exploration of OIP5-AS1 in EC cells, we carried out FISH assay and the outcomes disclosed that the main distribution of OIP5-AS1 was in cytoplasm in EC cells (Fig. 2A). Therefore, we raised a hypothesis that OIP5-AS1 regulates EC cells through ceRNA mode. Then, we searched http://starBase.sysu.edu.cn/ for potential miRNAs containing potential binding sites with OIP5-AS1. Among all the candidates, miR-152-3p was linked to the endometrial receptivity status [15]. Therefore, we selected miR-152-3p for the followingup experiments. After the transfection with sh-NC and sh-OIP5-AS1\#1, RT-qPCR measured the expression of 


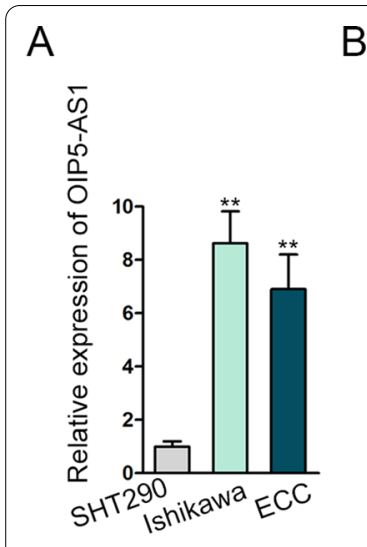

D

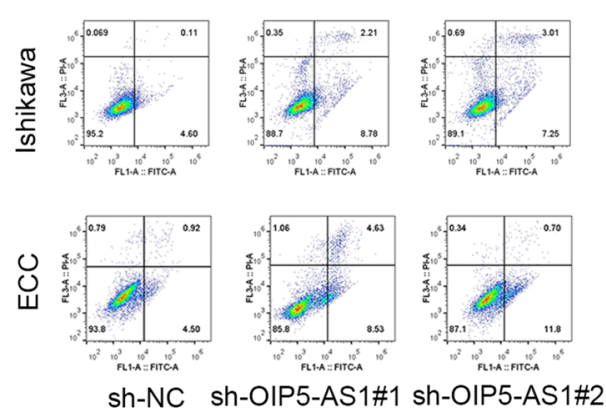

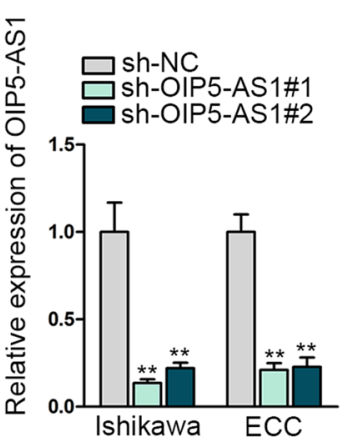
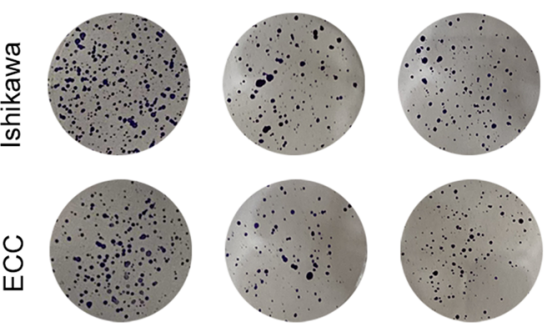

sh-NC sh-OIP5-AS1\#1 sh-OIP5-AS1\#2

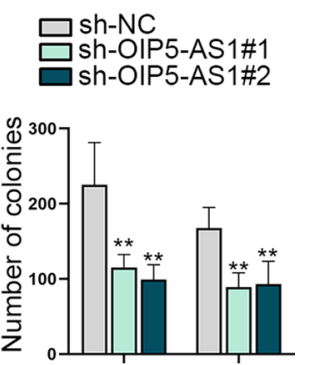

Ishikawa ECC

$E$
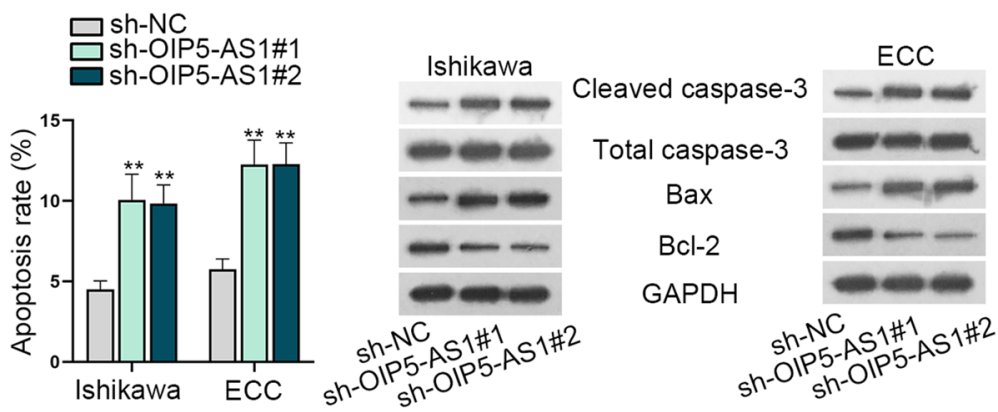

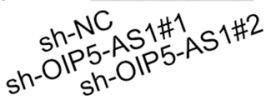

$\mathrm{F}$
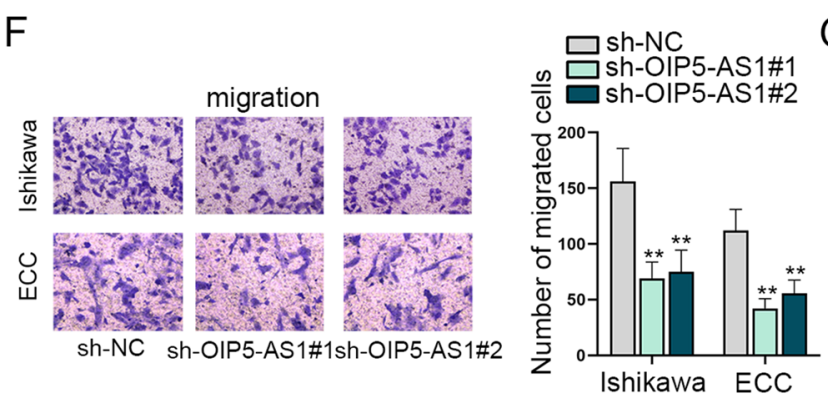

G
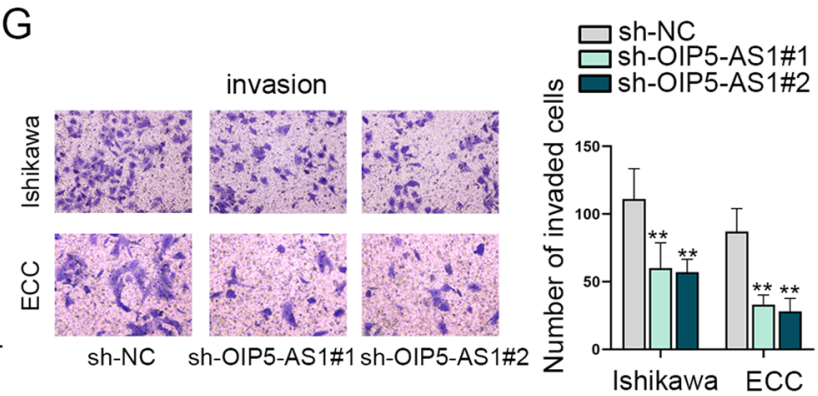

$\mathrm{H}$

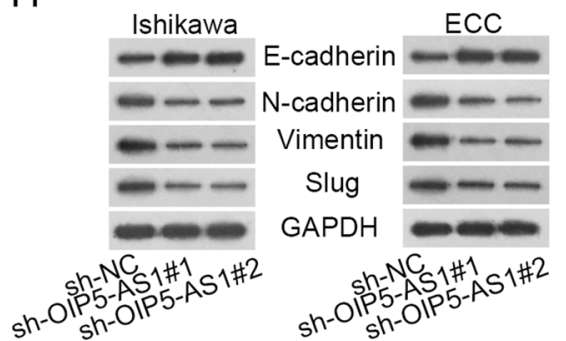

Fig. 1 OIP5-AS1 isup-regulated in EC cells and boosted the development of EC. A OIP5-AS1 in Ishikawa and ECC cells by RT-qPCR. B Efficacy assessment of OIP5-AS1 silence through RT-qPCR in Ishikawa and ECC cells. C Cell proliferative ability evaluation in OIP5-AS1-ablated Ishikawa and ECC cells through the implementation of colony formation assays. D Cell apoptosis evaluation in transfected cells through flow cytometry. (E) Apoptosis-related proteins in transfected cells via western blot. F, G Cell migration and invasion assessment in transfected cells through transwell assays. (H) Estimate of associated proteins of EMT by western blot in transfected cells. ${ }^{* *} \mathrm{P}<0.01$ 


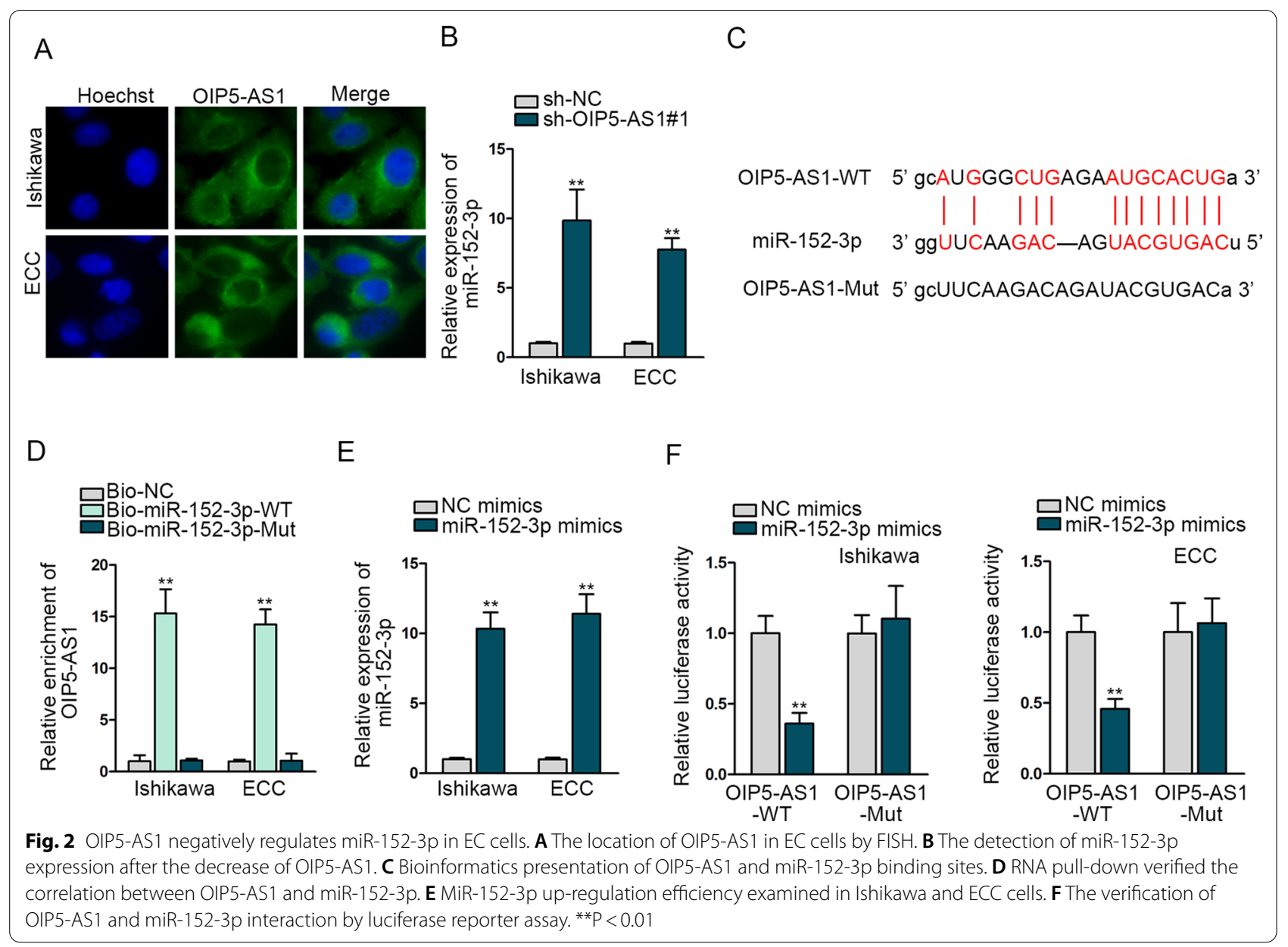

miR-152-3p in Ishikawa and ECC cells. Data revealed that miR-152-3p expression was escalated significantly (Fig. 2B), showing that OIP5-AS1 expression had a negative correlation with miR-152-3p expression. The binding sequences between miR-152-3p and OIP5-AS1 were presented in Fig. 2C. Subsequently, RNA pull down showed that biotinylated miR-152-3p-WT, instead of miR-1523p-Mut, could pull down OIP5-AS1 into sediment, proving their interaction (Fig. 2D). Consequences of RTqPCR delineated that miR-152-3p expression was elevated in response to miR-152-3p mimics (Fig. 2E). Data of luciferase reporter assays manifested that miR-152-3p up-regulation could dwindle the luciferase activity of plasmid with OIP5-AS1-WT instead of OIP5-AS1-Mut, further verifying the combination (Fig. 2F). In a word, OIP5-AS1 negatively regulates miR-152-3p in EC cells.

\section{Up-regulation of miR-152-3p suppresses the process of EC cells}

We designed functional assays to attest the biological functions of miR-152-3p in EC cells. Results of colony formation disclosed that miR-152-3p overexpression repressed the proliferation of EC cells (Fig. 3A). Moreover, miR-152-3p up-regulation lifted the apoptosis rate of EC cells (Fig. 3B). The proteins of apoptosis were changed in answer to miR-152-3p up-regulation as well (Fig. 3C). The migratory and invasive capacities were attenuated in transwell assays due to miR-152-3p overexpression (Fig. 3D, E). EMT of EC cells was suppressed after the overexpression of miR-152-3p as evidenced by western blot (Fig. 3F). In conclusion, miR-152-3p up-regulation hinders the process of EC cells.

\section{OIP5-AS1 contributes to the growth of EC cells via down-regulating miR-152-3p expression}

To study how OIP5-AS1 regulated miR-152-3p to affect the EC progression, we performed the rescue assays. MiR-152-3p inhibitor was transfected into cells for efficiency analysis (Fig. 4A). Next, we conducted a series of rescue experiments. The declined proliferative capacities imposed by OIP5-AS1 knockdown were enhanced by miR-152-3p down-regulation (Fig. 4B). The increased apoptosis induced by OIP5-AS1 silence were offset via down-regulated miR-152-3p (Fig. 4C). 


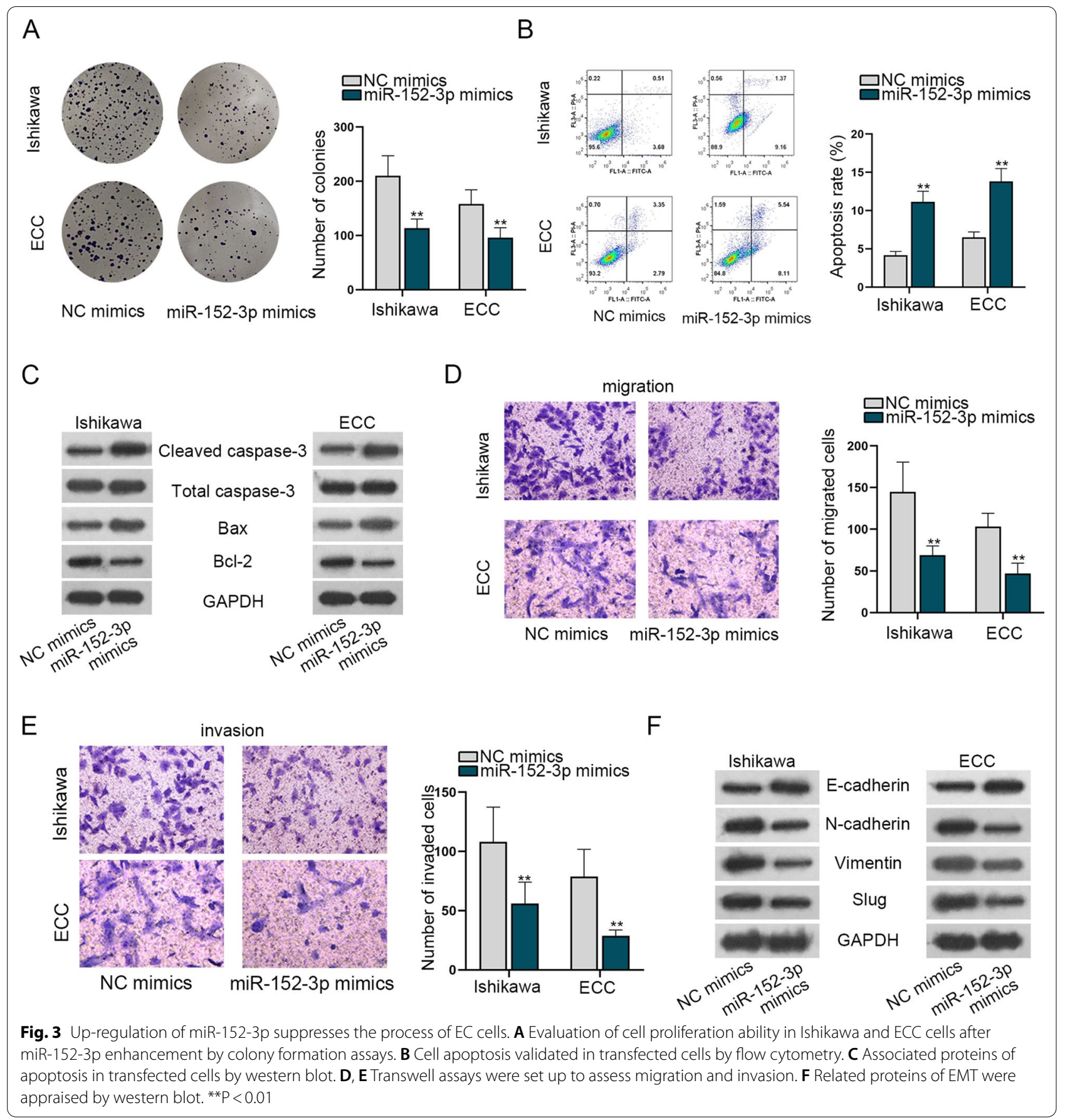

The elevated cleaved caspase- 3 and Bax proteins along with decreased $\mathrm{Bcl}-2$ proteins, which were caused by OIP5-AS1 knockdown, were countervailed by miR152-3p depletion (Fig. 4D). In terms of transwell assays, down-modulated OIP5-AS1 dropped the capacities of EC cells to migrate and invade, which could be restored by miR-152-3p inhibitor (Fig. 4E, F). The relevant proteins of EMT were changed due to OIP5-AS1 downregulation. After miR-152-3p inhibitor was transfected into Ishikawa and ECC cells, this trend was reversed (Fig. 4G). In brief, OIP5-AS1 facilitates the growth of EC cells through decreasing miR-152-3p expression. 


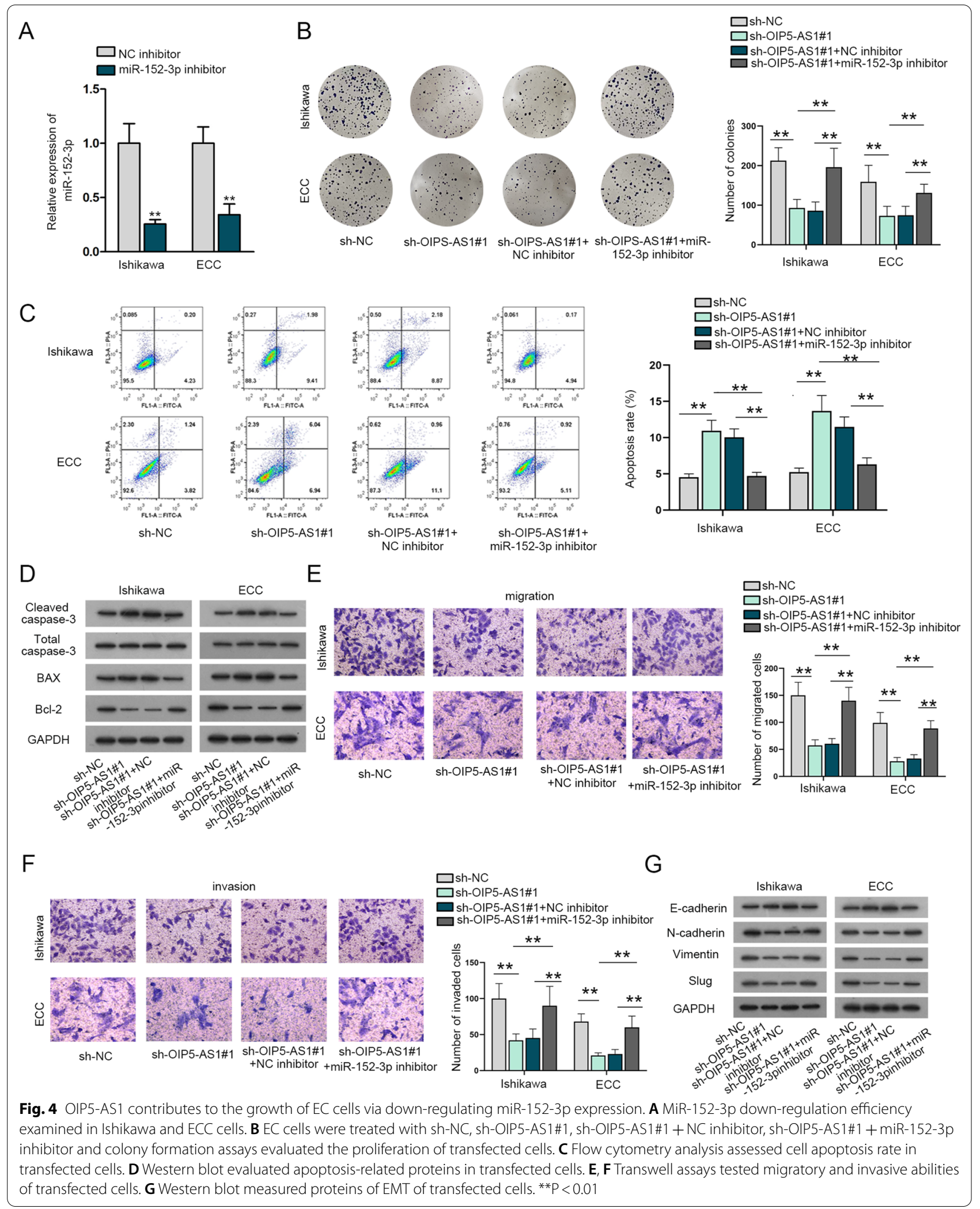


SLC7A5 overexpression can rescue the influences of OIP5-AS1 down-regulation on EC cell progression

It is well recognized that the downstream targetsmRNAs could be translated into proteins to exert functions on the diseases. We searched starBase and found NPTN, IRS1, SLC7A5, IGF1R and POLDIP2 had latent binding sites with miR-152-3p. These mRNAs expressions were tested in miR-152-3p-overexpressed Ishikawa and ECC cells. Data showed that only SLC7A5 expression was lessened overtly while no changes could be seen in other mRNAs (Fig. 5A). The binding sites between miR152-3p and SLC7A5 were exhibited in Fig. 5B. Results of RIP portrayed the coexistence of SLC7A5, OIP5-AS1 and miR-152-3p in RNA-induced silencing complexes (RISCs) (Fig. 5C). The luciferase activity of SLC7A5-WT group was undermined by miR-152-3p mimics, which was recovered by OIP5-AS1 overexpression. Considering the luciferase activity of SLC7A5-Mut group, no obvious changes were discovered (Fig. 5D). Additionally, we applied rescue assays to verify OIP5-AS1 modulated SLC7A5 to affect the progression of EC cells. Firstly, and the efficiency of pcDNA3.1/SLC7A5 was assessed in Ishikawa and ECC cells (Fig. 5E). The outcomes of rescue assays manifested that up-regulation of SLC7A5 could countervail the changes in proliferation (Fig. $5 \mathrm{~F}$ ), apoptosis (Fig. 5G, H), migration and invasion (Fig. 5I, J) as well as EMT of EC cells (Fig. 5K), which were induced through OIP5-AS1 knockdown. To sum up, OIP5-AS1 accelerates the growth of EC cells by up-regulating SLC7A5.

\section{Discussion}

According to previous researches, lncRNAs are regarded as underlying biomarkers in human cancers and non-tumors. For example, lncRNA ZNF180-2 was considered as a prognostic biomarker among clear cell renal cell carcinoma [16]. LncRNA PANDAR promoted proliferation, cell cycle in thyroid cancer cell [17]. LncRNA-TSI inhibited renal fibrogenesis through modulating the TGF- $\beta /$ Smad3 pathway negatively [18]. OIP5-AS1 was analyzed in lung adenocarcinoma [19]. Based on these precedent findings, the focus of this study is the function of OIP5-AS1 in EC. Intriguingly,
OIP5-AS1 was observed being highly expressed in EC cells. Accordingly, OIP5-AS1 down-regulation suppressed proliferative, migratory and invasive capacities, and EMT while fostering apoptosis in EC progression.

Lately, accumulating attention was attracted to competing endogenous RNA (ceRNA) system. Supporters believed that IncRNAs could function as targets of miRNAs so that their downstream targets could be liberated to translate into proteins [20]. For instance, RSU1P2 boosted the cervical cancer growth via serving as a ceRNA against let-7a [21]. XIST was reported to exert oncogenic function in colorectal cancer via targeting miR-132-3p [22]. In this study, we testified the subcellular localization of OIP5-AS1 in EC cells. OIP5-AS1 was affirmed as a target of miR-152-3p. In addition, the downstream target SLC7A5 was selected out to be negatively modulated by miR-152-3p. The competing relationship between OIP5-AS1 and SLC7A5 was confirmed by luciferase reporter assays. More importantly, OIP5-AS1 could boost the progression of EC cells via up-regulating SLC7A5.

Increasing evidence demonstrated the crucial effects of miRNAs on the development and progression of human tumors. Prior researches have validated that miR-152 worked as a tumor inhibitor in breast cancer via modulating PIK3CA [23]. MiR-152-3p modulated hepatic glycogenesis through targeting PTEN [24]. Moreover, miR-152-3p down-regulation could rescue the effects of HOTAIR silence on the process of malignant melanoma [25]. In our study, it was found that miR-152-3p could be negatively regulated by OIP5-AS1. Moreover, miR152-3p enhancement could restrict the growth of EC cells. Besides, miR-152-3p depletion could neutralize the influences imposed by OIP5-AS1 knockdown.

\section{Conclusions}

In summary, the data of our study disclosed that OIP5AS1 is able to foster the abilities of cell to proliferate, migrate and invade via targeting miR-152-3p to up-regulate SLC7A5 in EC cells, suggesting OIP5-AS1 might be a valuable target against $\mathrm{EC}$.

\footnotetext{
(See figure on next page.)
}

Fig. 5 SLC7A5 overexpression can rescue the effects of OIP5-AS1 down-regulation on the development of EC cells. A These mRNAs expressions were detected by RT-qPCR in cells after the overexpression of miR-152-3p. B Bioinformatics presentation of miR-152-3p and SLC7A5 binding sites. C RIP assay testified OIP5-AS1, miR-152-3p and SLC7A5 coexisted in RNA induced silencing complexes (RISCs). D Luciferase activity of SLC7A5-WT/Mut in response to NC mimics or miR-152-3p mimics in EC cells as well as the addition of pcDNA3.1-OIP5-AS1 into cells. E Overexpressed SLC7A5 was validated in RT-qPCR. The influence of sh-OIP5-AS1\#1 on EC cells proliferation $(\mathbf{F})$, apoptosis $(\mathbf{G}, \mathbf{H})$, and migration as well as invasion $(\mathbf{I}-J)$ and EMT $(\mathrm{K})$ was rescued by overexpression of SLC7A5. ${ }^{* *} \mathrm{P}<0.01$ 


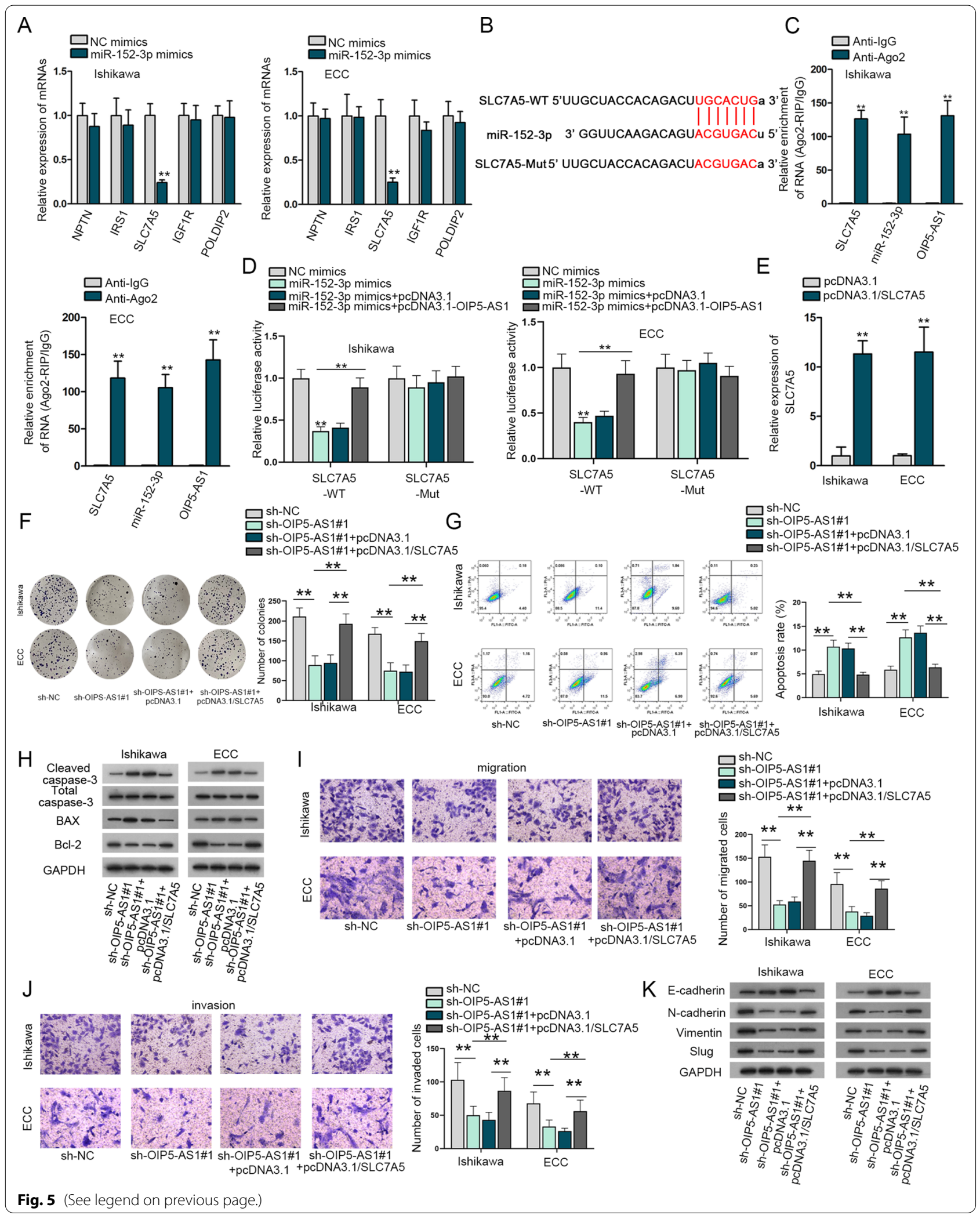




\section{Abbreviations}

EC: Endometrial carcinoma; IncRNAs: Long non-coding RNAs; EMT: Epithelialmesenchymal transition; miRNAs: MicroRNAs; URT: Untranslated region; RISCs: RNA-induced silencing complexes; ceRNA: Competing endogenous RNA; FBS: Fetal bovine serum; RT-qPCR: RNA extraction and real-time quantitative PCR; PBS: Phosphate-buffered saline; WT: Wild-type; Mut: Mutated; RIP: RNA immunoprecipitation.

\section{Supplementary Information}

The online version contains supplementary material available at https://doi. org/10.1186/s12935-021-02061-0.

Additional file 1: Figure S1. (A) Efficacy assessment of OIP5-AS1 overexpression via RT-qPCR in Ishikawa and ECC cells. (B) Detection of proliferation capacity of Ishikawa and ECC cells transfected with pcDNA3.1/OIP5AS1 or pcDNA3.1 via colony formation assays. (C) Apoptosis evaluation of transfected cells via flow cytometry assay. (D-E) Assessment of migration and invasion of transfected cells through the implementation of transwell assays. (F) Estimate of associated proteins of EMT in transfected cells by western blot. ${ }^{* *} \mathrm{P}<0.01$.

\section{Acknowledgements}

We appreciate all the participants who provide supports in the study.

\section{Authors' contributions}

In the process of revision, ML and JM prepared the figures, JM and HW prepared the data collection as well as analysis. CL, TL and JM contributed to the experiment. Moreover, they recorded the result. ML and JM prepared first draft and revised the manuscript. All authors read and approved the final manuscript.

\section{Funding}

None.

Availability of data and materials

Data were not shared.

\section{Declarations}

Ethics approval and consent to participate

Not applicable.

\section{Consent for publication}

Not applicable.

\section{Competing interests}

None.

Received: 22 November 2019 Accepted: 28 June 2021 Published online: 21 August 2021

\section{References}

1. López-Janeiro Á, Ruz-Caracuel I, Ramón-Patino JL, De Los Ríos V, Villalba Esparza M, Berjón A, Yébenes L, Hernández A, Masetto I, Kadioglu E, et al. Proteomic analysis of low-grade, early-stage endometrial carcinoma reveals new dysregulated pathways associated with cell death and cell signaling. Cancers. 2021;13:4.

2. Bartosch C, Pires M, Jeronimo C, Lopes JM. The role of pathology in the management of patients with endometrial carcinoma. Fut Oncol. 2017:13(11):1003-20.

3. Loukovaara M, Luomaranta A, Leminen A. Treatment of endometrial carcinoma. Duodecim. 2014;130(22-23):2348-55.

4. Abu Saadeh F, Langhe R, Galvin DM, Sa OT, O'Donnell DM, Gleeson N, Norris LA. Procoagulant activity in gynaecological cancer patients; the effect of surgery and chemotherapy. Thromb Res. 2016;139:135-41.
5. Quinn JJ, Chang HY. Unique features of long non-coding RNA biogenesis and function. Nat Rev Genet. 2016;17(1):47-62.

6. Zhang Z, Liu X, Xu H, Li P, Zeng T, Du W, Lu X. LINC01170 promotes the progression of endometrial carcinoma by activating the AKT pathway. J BUON. 2018;23(6):1745-52.

7. Zou MF, Ling J, Wu QY, Zhang CX. Long non-coding RNA PVT1 functions as an oncogene in ovarian cancer via upregulating SOX2. Eur Rev Med Pharmacol Sci. 2018;22(21):7183-8.

8. Dai J, Xu L, Hu X, Han G, Jiang H, Sun H, Zhu G, Tang X. Long noncoding RNA OIP5-AS1 accelerates CDK14 expression to promote osteosarcoma tumorigenesis via targeting miR-223. Biomed Pharmacother. 2018;106:1441-7.

9. Wang M, Sun X, Yang Y, Jiao W. Long non-coding RNA OIP5-AS1 promotes proliferation of lung cancer cells and leads to poor prognosis by targeting miR-378a-3p. Thoracic Cancer. 2018;9(8):939-49.

10. Zou Y, Yao S, Chen X, Liu D, Wang J, Yuan X, Rao J, Xiong H, Yu S, Yuan X, et al. LncRNA OIP5-AS1 regulates radioresistance by targeting DYRK1A through miR-369-3p in colorectal cancer cells. Eur J Cell Biol. 2018;97(5):369-78.

11. Mishra S, Yadav T, Rani V. Exploring miRNA based approaches in cancer diagnostics and therapeutics. Crit Rev Oncol Hematol. 2016;98:12-23.

12. Wang Y, Zhu Y, LV P, Li L. The role of miR-21 in proliferation and invasion capacity of human tongue squamous cell carcinoma in vitro. Int J Clin Exp Pathol. 2015;8(5):4555-63.

13. Jin L, Yi J, Gao Y, Han S, He Z, Chen L, Song H. MiR-630 inhibits invasion and metastasis in esophageal squamous cell carcinoma. Acta Biochim Biophys Sin. 2016:48(9):810-9.

14. Yue Z, Shen JJ, Huang QT, Qin YF, Li XN, Liu GB. MiR-135b promotes proliferation of endometrial carcinoma cells by targeting FOXO1. Nan fang yi ke da xue xue bao J Southern Med Univ. 2016;36(5):675-80.

15. Drissennek L, Baron C, Brouillet S, Entezami F, Hamamah S, Haouzi D. Endometrial miRNome profile according to the receptivity status and implantation failure. Hum Fert. 2020;7:1-13.

16. Ellinger J, Alam J, Rothenburg J, Deng M, Schmidt D, Syring I, Miersch H, Perner S, Muller SC. The long non-coding RNA Inc-ZNF180-2 is a prognostic biomarker in patients with clear cell renal cell carcinoma. Am J Cancer Res. 2015;5(9):2799-807.

17. Li Z, Gao B, Hao S, Tian W, Chen Y, Wang L, Zhang X, Luo D. Knockdown of IncRNA-PANDAR suppresses the proliferation, cell cycle and promotes apoptosis in thyroid cancer cells. Excli J. 2017;16:354-62.

18. Wang P, Luo ML, Song E, Zhou Z, Ma T, Wang J, Jia N, Wang G, Nie S, Liu Y, et al. Long noncoding RNA Inc-TSI inhibits renal fibrogenesis by negatively regulating the TGF-beta/Smad3 pathway. Sci Transl Med. 2018;10:462.

19. Deng J, Deng H, Liu C, Liang Y, Wang S. Long non-coding RNA OIP5-AS functions as an oncogene in lung adenocarcinoma through targeting miR-448/BCl-2. Biomed Pharmacother. 2018;98:102-10.

20. Li Z, Wu X, Gu L, Shen Q, Luo W, Deng C, Zhou Q, Chen X, Li Y, Lim Z, et al. Long non-coding RNA ATB promotes malignancy of esophageal squamous cell carcinoma by regulating miR-200b/Kindlin-2 axis. Cell Death Dis. 2017;8(6):e2888.

21. Liu Q, Guo X, Que S, Yang X, Fan H, Liu M, Li X, Tang H. LnCRNA RSU1P2 contributes to tumorigenesis by acting as a ceRNA against let-7a in cervical cancer cells. Oncotarget. 2017;8(27):43768-81.

22. Song H, He P, Shao T, Li Y, Li J, Zhang Y. Long non-coding RNA XIST functions as an oncogene in human colorectal cancer by targeting miR132-3p. J BUON. 2017:22(3):696-703.

23. Ge S, Wang D, Kong Q, Gao W, Sun J. Function of miR-152 as a tumor suppressor in human breast cancer by targeting PIK3CA. Oncol Res. 2017;25(8):1363-71.

24. Wang S, Wang L, Dou L, Guo J, Fang W, Li M, Meng X, Man Y, Shen T, Huang $X$, et al. MicroRNA 152 regulates hepatic glycogenesis by targeting PTEN. FEBS J. 2016;283(10):1935-46.

25. Luan W, Li R, Liu L, Ni X, Shi Y, Xia Y, Wang J, Lu F, Xu B. Long non-coding RNA HOTAIR acts as a competing endogenous RNA to promote malignant melanoma progression by sponging miR-152-3p. Oncotarget. 2017;8(49):85401-14.

\section{Publisher's Note}

Springer Nature remains neutral with regard to jurisdictional claims in published maps and institutional affiliations. 